\title{
Morbidity and Mortality in Patients with Perioperative COVID- 19 Infection: Prospective Cohort in General, Gastroesophagic, Hepatobiliary, and Colorectal Surgery
}

\author{
Martin Inzunza ${ }^{1,2}$ (10 $\cdot$ Cecilia Romero ${ }^{1,2} \cdot$ María Jesús Irarrázaval $^{2} \cdot$ Magdalena Ruiz-Esquide $^{2} \cdot$ \\ Pablo Achurra $^{1,2} \cdot$ Nicolás Quezada $^{1,2} \cdot$ Fernando Crovari $^{1,2} \cdot$ Rodrigo Muñoz $^{1,2}$
}

Accepted: 6 March 2021/Published online: 21 March 2021

(C) Société Internationale de Chirurgie 2021

\begin{abstract}
Background Severe acute respiratory syndrome due to coronavirus 2 has rapidly spread worldwide in an unprecedented pandemic. Patients with an ongoing COVID-19 infection requiring surgery have higher risk of mortality and complications. This study describes the mortality and morbidity in patients with perioperative COVID-19 infection undergoing elective and emergency surgeries.

Methods Prospective cohort of consecutive patients who required a general, gastroesophageal, hepatobiliary, colorectal, or emergency surgery during COVID-19 pandemic at an academic teaching hospital. The primary outcome was 30-day mortality and major complications. Secondary outcomes were specific respiratory mortality and complications.

Results A total of 701 patients underwent surgery, 39 (5.6\%) with a perioperative COVID-19 infection. 30-day mortality was $12.8 \%$ and $1.4 \%$ in patients with and without COVID-19 infection, respectively $(p<0.001)$. Major surgical complications occurred in $25.6 \%$ and $6.8 \%$ in patients with and without COVID-19 infection, respectively $(p<0.001)$. Respiratory complications occurred in $30.8 \%$ and $1.4 \%$ in patients with and without COVID-19 infection, respectively $(p<0.001)$. Mortality due to a respiratory complication was $100 \%$ and $11.1 \%$ in patients with and without COVID-19 infection, respectively $(p<0.006)$.

Conclusions 30-day mortality and surgical complications are higher in patients with perioperative COVID-19 infection. Indications for elective surgery need to be reserved for non-deferrable procedures in order to avoid unnecessary risks of non-urgent procedures.
\end{abstract}

\section{Introduction}

Due to coronavirus 2 (SARS-CoV-2), a severe acute respiratory syndrome has rapidly spread globally since the beginning of 2020, affecting most countries in what has become an unprecedented pandemic. The World Health

Rodrigo Muñoz

rmunozc@med.puc.cl

1 Department of Digestive Surgery, Pontificia Universidad Católica de Chile, Santiago, Chile

2 School of Medicine, Pontificia Universidad Católica de Chile, Santiago, Chile
Organization (WHO) declared the COVID-19 pandemic on March 11, 2020. A few days later, Chile declared phase 4 of the pandemic, establishing measures designed to limit the spread of the virus. To focus on human resources and infrastructure, public and private health institutions suspended or postponed elective surgeries, prioritizing patients with surgical emergencies or oncological diseases [1]. By the end of August in Chile, 447,527 COVID-19 cases have been diagnosed, with a rate of 2300 per 100,000 inhabitants [2]. In our center, from March to August, a total of 1098 hospitalized patients have tested positive for COVID-19, with 925 discharges (84\%) and 38 patients that remained hospitalized (3.5\%) on August 31. In our 
institution, a total of 135 patients have died due to COVID$19(12.3 \%)$, of which $67 \%(n=90)$ were older than 70 years. Of the hospitalized patients, 23 patients remained in the critical care unit.

Patients undergoing surgery have a greater risk of becoming infected with COVID-19 due to the surgery's pro-inflammatory nature and its associated immunosuppressive response. Mechanical ventilation's deleterious effect facilitates the greater risk of respiratory complications during surgery [3, 4]. Studies have shown an increased risk of complications and mortality in patients with COVID-19 infection undergoing surgery throughout this pandemic. To date, the international collaborative group CovidSurg has the most extensive available data on complications in patients undergoing surgery during this pandemic. The results from 1128 patients from 235 hospitals in 24 countries have shown a high 30-day mortality rate of $23.8 \%$. Respiratory complications occurred in $51.2 \%$ of the patients, with a mortality of $38 \%$, which represents $82.6 \%$ of all deaths in the study [5]. Other series have also shown high mortality rates between 20 and $25 \%$, with respiratory complications been reported in up to $40-50 \%$ of patients [6-15].

The objective of this study is to report 30-day mortality early morbidity, pulmonary complications, and mortality in patients who underwent emergency or elective surgery in our Department of General and Digestive Surgery, including gastroesophageal, HBP, and colorectal surgeries, since the beginning of the pandemic outbreak in Chile.

\section{Methods}

\section{Study design}

A prospective observational cohort study was conducted at the Hospital Clínico Pontificia Universidad Católica, Chile. The institutional ethics committee approved the present study.

\section{Patient selection}

All patients $\geq 15$ years old who underwent elective or emergency abdominal surgeries consecutively from March to August of 2020 were included. Esophagogastric, hepatobiliary, colorectal, and general surgery procedures were included.

\section{COVID diagnostic test}

COVID-19 infection was determined based on the results of viral SARS-CoV-2 RNA detection by quantitative RTPCR. Samples were obtained through nasopharyngeal swabs according to the standardized technique. COVID-19 PCR has a specificity close to $100 \%$; however, the sensitivity varies depending on the viral load, the site of sample collection, and the moment that the sample is obtained in the evolution of the disease, it means a lower sensitivity in the first days of infection and higher after seven days of infection [16].

Perioperative infection was defined in all patients with SARS-CoV-2 disease diagnosed within seven days before and 30 days after surgery. For elective patients, the sample was obtained up to $48 \mathrm{~h}$ before surgery. A sample was obtained immediately before the transfer to the OR for patients undergoing emergency surgery with a previous negative PCR test or unknown COVID status. During the 30-day follow-up, PCR tests were indicated if the patient developed fever or respiratory symptoms.

\section{Clinical variables and follow-up}

Relevant clinical variables included age, sex, comorbidities, body mass index, type of surgery (elective vs. emergency), surgical approach (open vs. laparoscopic), type of anesthesia, hospital length of stay, morbidity, and mortality. Morbidity was categorized according to the ClavienDindo classification [17]. Postoperative follow-up was carried out until 30 days after surgery and included outpatient visits, telemedicine, and telephone calls [18].

\section{Primary and secondary outcomes}

The primary outcomes were 30-day mortality and major complications. Major complications were defined as Clavien-Dindo score $\geq$ III. Secondary outcomes included specific respiratory mortality and respiratory complications, defined as pneumonia, respiratory distress, need for non-invasive mechanical ventilation, or invasive mechanical ventilation (IMV). Reoperations and readmissions rates during the 30-days follow-up were also analyzed.

A relative risk (RR) analysis was performed for both primary outcomes, with a $95 \%$ confidence interval.

\section{Institutional protocols}

The institutional protocols adopted for the care of patients undergoing emergency or elective surgical procedures with unknown or confirmed COVID-19 infection, from arrival to the emergency service, ward, and postoperative recovery, are detailed in Table 1 [19]. The protocol includes COVID-19 sample collection, admission to a specific ward for COVID-19 (+) patients, restriction of healthcare personnel, avoiding unnecessary flow in the ward, pneumoperitoneum evacuation protocol, and differentiated sample transport. For COVID-19 (+) patients or patients 
Table 1 Institutional protocols adopted for the care of patients undergoing surgical procedures with unknown or confirmed COVID-19 infection

Operation room (OR) preparation

Use the same assigned OR

Minimum of people in the OR

Only necessary items for surgery

The use of cell phones is prohibited inside the OR

Maintain the OR with positive pressure

Handling of supplies outside the OR

Ensure a filter presence in the expiratory tract and between the tube and the circuit

Use signs to indicate contact and droplet caution

Ask for the patient once the OR is ready for expedited access

Patients' transfer should always be with a mask

Patient management in the $O R$

Environmental management during anesthetic induction: doors closed, access limitation, preferential use of disposable materials

Surfaces cleaning using quaternary ammonium

The anesthesia team must use an N95 mask, face shield or googles, disposable cap and shoe covers, disposable plastic long sleeve bib, and procedure gloves

The surgery team must use scrub clothing, disposable plastic bib, N95 mask, face shield or googles, disposable cap and shoe covers

Ending the procedure

Patients confirmed or suspected with COVID-19 destined to low complexity services must accomplish recovery time in the OR

Patients with ICU requirements are immediately transferred with the same personal team to a previously reserved bed and with the respective isolation

Surgery, anesthesia and nursing teams must remove their PPE inside the OR using procedure gloves and a subsequent hand wash

Disinfection of face shields and googles with quaternary ammonium

Minimally invasive procedures

Skin incision according to trocar diameter to avoid $\mathrm{CO} 2$ leakage by counter-opening

Intraabdominal pressure as minimum as possible. Recommended $10-12 \mathrm{mmHg}$

Use of a safe filter aspiration/exufflation system with a Luer-lock connection to the pneumoperitoneum

Pneumoperitoneum safe evacuation and at the moment of piece extraction

To avoid the retrograde passage of gas into the system, the insufflating trocar valve was closed before disconnection or turning off the insufflation at the end of the surgery

Pneumoperitoneum should be wholly evacuated through the filter safe aspiration/exsufflation system with all the trocars in position and before its removal

Extraction of the piece following the complete evacuation of $\mathrm{CO} 2$ and gas

Use of surgical drainage only if mandatory

Aponeurosis closure after exufflation

Avoid using automatic closing devices

Consider the open approach if any of the above requirements cannot be accomplished

with pending PCR results at the time of surgery, they were transferred to a special recovery unit and then to a differentiated basic ward. After surgery, patients with ICU requirements were transferred to a differentiated ICU unit exclusive for patients with COVID-19 infection.

\section{Statistical analysis}

Statistical analysis was performed with SPSS 25 (IBM Corp., Amonk, New York). Patients with COVID-19 infection and without COVID-19 were compared with Student's $t$-test or Mann-Whitney test depending on data distribution, and chi-square test for comparison of proportions. All data were expressed as mean and standard 
deviation or median and interquartile range. Differences were considered significant when $p$ value $<0.05$.

\section{Results}

From March to August of 2020, during the ongoing COVID-19 outbreak in Chile, 701 patients underwent a general, esophagogastric, hepatobiliary, or coloproctological surgery at our university teaching affiliated hospital. The mean age of patients was $51 \pm 18$ years, and 343 (49\%) were women. At least one comorbidity at the time of the surgery was found in $50.1 \%$ of patients (Table 2). 124 out of 701 surgeries were oncological procedures $(17.7 \%$, Table 2). Perioperative SARS-CoV-2 infection was detected in 39 patients $(5.6 \%)$, in 20 of them $(51.3 \%)$ were detected before surgery (Fig. 1).

Most of the procedures $(368,52.5 \%)$ were surgical emergencies, which were more frequent in patients with COVID-19 infection (71.8\% COVID-19 (+) vs. $51.4 \%$ COVID-19 (-), $p=0.03$ ) (Table 3). General anesthesia was used in $653(93.2 \%)$ of surgeries. A total of $463(66 \%)$ surgeries were performed by a minimally invasive approach, either laparoscopic and/or endoscopic, under general anesthesia (ERCP, rendezvous procedure, others). The three most common surgeries performed were 191 (27.2\%) cholecystectomies, 129 (18.4\%) appendectomies, and $90(12.8 \%)$ colorectal surgeries (Table 3$)$.

Overall 30-day mortality was $2 \%(\mathrm{n}=14)$. However, in patients with COVID-19 infection, 30-day mortality was higher $(12.8 \%(5 / 39)$ COVID-19 $(+)$ versus. $1.4 \%(9 / 662)$

Table 2 Demographic variables

\begin{tabular}{|c|c|c|c|c|}
\hline & Total & COVID-19 (+) & COVID-19 (-) & $p$ value \\
\hline Patients & 701 & 39 & 662 & - \\
\hline \multicolumn{5}{|l|}{ Age; mean $\pm S D$} \\
\hline Years & $51 \pm 18.4$ & $46 \pm 19.4$ & $52 \pm 18.4$ & 0.124 \\
\hline \multicolumn{5}{|l|}{$\operatorname{Sex}(\%)$} \\
\hline Male & $358(51 \%)$ & $19(48.7 \%)$ & $339(51.2 \%)$ & \multirow[t]{2}{*}{0.890} \\
\hline Female & $343(49 \%)$ & $20(51.3 \%)$ & $323(48.8 \%)$ & \\
\hline \multicolumn{5}{|l|}{$A S A ; n(\%)$} \\
\hline I-II & $649(92.6 \%)$ & $34(87.2 \%)$ & $615(92.9 \%)$ & \multirow[t]{2}{*}{0.308} \\
\hline$\geq \mathrm{III}$ & $52(7.4 \%)$ & $5(12.8 \%)$ & $47(7.1 \%)$ & \\
\hline \multicolumn{5}{|l|}{$B M I ; n(\%)$} \\
\hline$\leq 18,5$ & $16(2.3 \%)$ & $3(7.7 \%)$ & $13(2 \%)$ & \multirow[t]{5}{*}{0.043} \\
\hline $18,5-24,9$ & $257(36.7 \%)$ & $15(38.5 \%)$ & $242(36.6 \%)$ & \\
\hline $25-29,9$ & $275(39.2 \%)$ & $10(25.6 \%)$ & $265(40 \%)$ & \\
\hline $30-39,9$ & $139(19.8 \%)$ & $11(28.2 \%)$ & $128(19.3 \%)$ & \\
\hline$\geq 40$ & $14(2 \%)$ & $0(0 \%)$ & $14(2.1 \%)$ & \\
\hline \multicolumn{5}{|l|}{ Comorbidities; $n(\%)$} \\
\hline None & $350(49.9 \%)$ & $19(48.7 \%)$ & $331(50 \%)$ & \multirow[t]{2}{*}{0.876} \\
\hline One or more & $351(50.1 \%)$ & $20(51.3 \%)$ & $331(50 \%)$ & \\
\hline Hypertension & $174(24.8 \%)$ & $11(28.2 \%)$ & $163(24.6 \%)$ & 0.615 \\
\hline Metabolic disease* & $96(13.7 \%)$ & $5(12.8 \%)$ & $91(13.7 \%)$ & 0.870 \\
\hline Respiratory disease & $45(6.4 \%)$ & $3(7.7 \%)$ & $42(6.3 \%)$ & 0.738 \\
\hline Cardiovascular disease & $46(6.6 \%)$ & $3(7.7 \%)$ & $43(6.5 \%)$ & 0.769 \\
\hline Neurological disease & $17(2.4 \%)$ & $1(2.6 \%)$ & $16(2.4 \%)$ & 0.953 \\
\hline Oncological $* *$ or immunosuppression & $57(8.1 \%)$ & $5(12.8 \%)$ & $52(7.9 \%)$ & 0.271 \\
\hline \multicolumn{5}{|l|}{ Cancer***, $n(\%)$} \\
\hline Yes & $124(17.7 \%)$ & $8(20.5 \%)$ & $116(17.5 \%)$ & \multirow[t]{2}{*}{0.634} \\
\hline No & $577(82.3 \%)$ & $31(79.5 \%)$ & $546(82.5 \%)$ & \\
\hline
\end{tabular}

$S D$ standard deviation, ASA American society of anesthesia classification, BMI body mass index.

*Includes diabetes, hypothyroidism, obesity, or dyslipidemia. **Includes cancer diagnosis not related to the surgical indication. ***Includes cancer diagnosis related to the surgical indication 


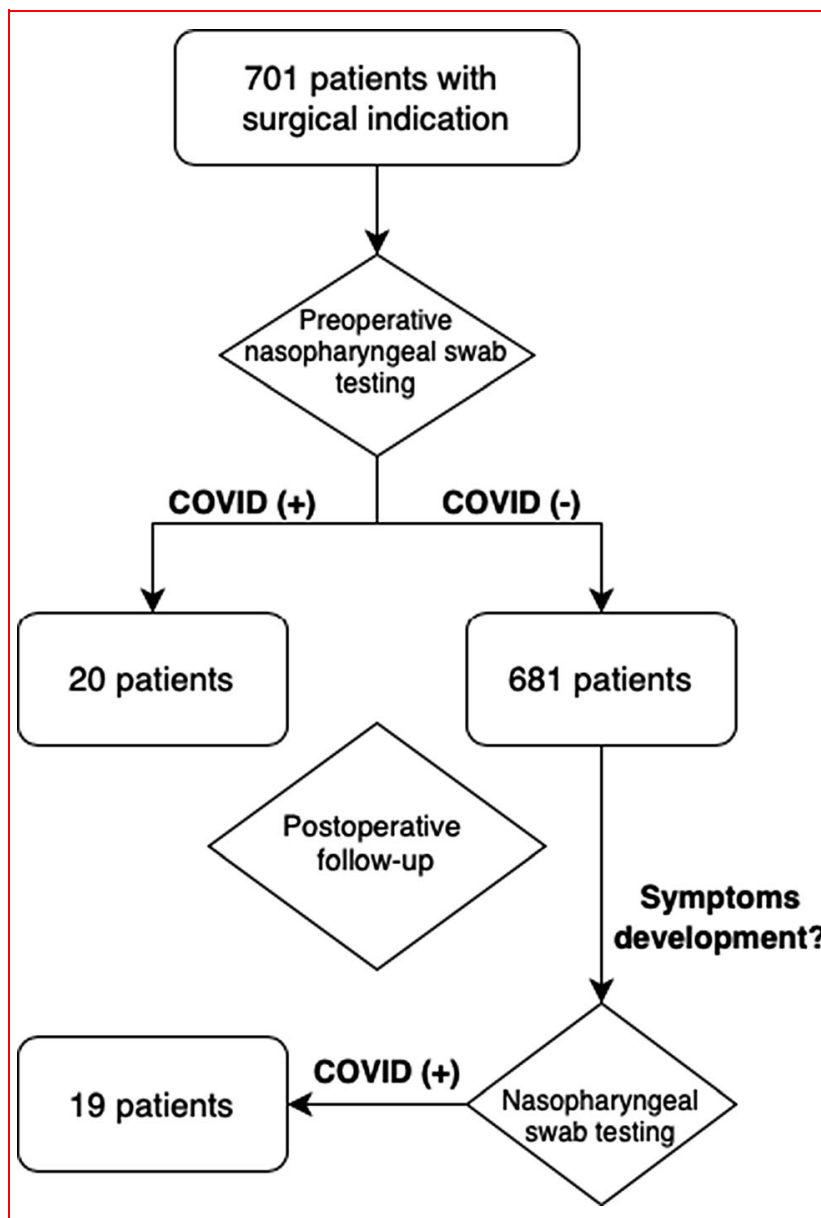

Fig. 1 The COVID-19 detection process

COVID-19 $(-), p<0.001)$ (Table 4). Among patients with COVID-19 (+) infection who died, in all of them, the cause of death was a consequence of a respiratory complication. In contrast, in patients without COVID-19 infection, a respiratory complication was the cause of death only in $11.1 \%(1 / 9)$ of patients $(100 \%(5 / 5)$ COVID-19 $(+)$ vs. $11.1 \%$ (1/9) COVID-19 $(-), p=0.006)$. Baseline characteristics of the five patients with mortality in the COVID-19 $(+)$ group are described in Table 5.

Major complications occurred in $7.9 \%(n=55)$ of patients and were more common in patients with COVID19 infection $(25.6 \%(10 / 39)$ COVID-19 $(+)$ versus $6.8 \%$ (45/662) COVID-19 (-), $p<0.001)$. Respiratory complications were more frequent in patients with COVID-19 infection (30.8\% (12/39) COVID-19 (+) versus 1.4\% (9/ 662) COVID-19 (-), $p<0.001)$. No difference was observed in terms of need of invasive mechanical ventilation (Table 4).

In the subgroup analysis of the COVID-19 (+) patients, 20 had a preoperative diagnosis and 19 were diagnosed after surgery. Among the 20 patients diagnosed before surgery, seven were symptomatic and 13 asymptomatic. Five patients in the symptomatic group developed complications. No morbidity was reported in the asymptomatic patients. Of the 19 patients diagnosed after surgery, all were symptomatic and 13 developed postoperative complications. Postoperative outcomes in the COVID-19 (+) group are detailed in Table 6 .

In the whole series, 31 patients $(4.4 \%)$ required an unplanned reoperation, without differences between both groups (5.1\% (2 of 39) COVID-19 (+) vs 4.4\% (29 of 662) COVID-19 (-), $p=0.825)$ ) (Table 4). The causes of reoperations in the COVID-19 (+) group were an exploratory laparoscopy after adhesiolysis in a patient with a small bowel obstruction and a postoperative abscess drainage.

During 30-day follow-up after hospital discharge, 34 (4.9\%) patients were readmitted. The cause of hospital readmission was surgical in $18(52.9 \%)$ and medical in 16 (47.1\%) patients. No differences were observed between patients with and without COVID-19 infection (Table 4).

One hundred and twenty-four patients $(17.7 \%)$ had an oncological diagnosis; among them, SARS-CoV-2 infection was detected in eight patients $(20.5 \%)$. One patient $(0.8 \%)$ with a sigmoid adenocarcinoma and severe COVID pneumonia got his surgery delayed.

In the RR analyses, patients with COVID-19 infection had a 9.4 times higher risk of dying after surgery than patients without COVID-19 infection (RR 9.43 [95\% CI 3.31-26.79], $p<0.001$ ). Excluding patients with postoperative COVID-19 infection, patients with preoperative COVID-19 infection had 7.3 times the risk of dying after surgery than patients without COVID-19 infection (RR 7.35 [95\% CI 1.69-31.86], $p=0.007$ ) (Table 7). Concerning surgical complications, patients with COVID-19 infection had 3.7 times the risk to develop a major complication after surgery than patients without COVID-19 infection (RR 3.77 [95\% CI 2.06-6.90], $p<0.001$ ). However, when patients with postoperative COVID-19 infection are excluded from the analysis, no differences are observed regarding to risk of major complication between patients with and without COVID-19 infection (RR 1.47 [95\% CI 0.38-5.64], $p=0.573$ ) (Table 7).

\section{Discussion}

It has been reported that patients undergoing surgery during the ongoing COVID-19 pandemic are at increased risk of complications and mortality. This study sought to report 30-day perioperative outcomes in patients undergoing emergency and oncological surgeries during the COVID19 outbreak at our university hospital center in Chile. We found that patients with SARS-CoV-2 infection had higher 
Table 3 Operative variables

\begin{tabular}{|c|c|c|c|c|}
\hline & Total & COVID-19 (+) & COVID-19 (-) & $p$ value \\
\hline Patients & 701 & 39 & 662 & - \\
\hline \multicolumn{5}{|c|}{ Urgency of surgery; $n(\%)$} \\
\hline Emergency & $368(52.5 \%)$ & $28(71.8 \%)$ & $340(51.4 \%)$ & \multirow[t]{2}{*}{0.030} \\
\hline Elective & $333(47.5 \%)$ & $11(28.2 \%)$ & $322(48.6 \%)$ & \\
\hline \multicolumn{5}{|c|}{ More frequent surgeries; $n(\%)$} \\
\hline Cholecystectomy & $191(27.2 \%)$ & $10(25.6 \%)$ & $181(27.3 \%)$ & \multirow[t]{6}{*}{0.625} \\
\hline Appendectomy & $129(18.4 \%)$ & $9(23.1 \%)$ & $120(18.1 \%)$ & \\
\hline Colorectal surgery & $90(12.8 \%)$ & $3(7.7 \%)$ & $87(13.1 \%)$ & \\
\hline Proctological surgery & $68(9.7 \%)$ & $3(7.7 \%)$ & $65(9.8 \%)$ & \\
\hline Hepatobiliary surgery & $57(8.1 \%)$ & $4(10.3 \%)$ & $53(8 \%)$ & \\
\hline Exploratory laparotomy & $49(7 \%)$ & $5(12.8 \%)$ & $44(6.6 \%)$ & \\
\hline \multicolumn{5}{|l|}{ Surgical approach; $n(\%)$} \\
\hline Open & $238(34 \%)$ & $11(28.2 \%)$ & $227(34.3 \%)$ & \multirow[t]{2}{*}{0.435} \\
\hline Minimally invasive* & $463(66 \%)$ & $28(71.8 \%)$ & $435(65.7 \%)$ & \\
\hline \multicolumn{5}{|l|}{ Anesthesia; $n(\%)$} \\
\hline General & $653(93.2 \%)$ & $36(92.3 \%)$ & $617(93.2 \%)$ & \multirow[t]{2}{*}{0.744} \\
\hline Local/regional & $48(6.8 \%)$ & $3(7.7 \%)$ & $45(6.8 \%)$ & \\
\hline \multicolumn{5}{|c|}{ Length of stay; median (IQR) } \\
\hline Days & $2(1-4)$ & $2(1-10)$ & $2(1-4)$ & 0.020 \\
\hline
\end{tabular}

$I Q R$ interquartile range.

*Laparoscopic and/or endoscopic under general anesthesia

mortality and severe surgical complications. Patients with COVID-19 infection also had a higher risk of respiratory complications, which was the cause dead in all patients with COVID-19 infection who died. Our results confirmed the high risk of mortality and complications in patients undergoing surgery during the COVID-19 pandemic.

Our results are in concordance with those reported in other studies [5-15], indicating that patients with COVID19 infection undergoing surgery are at higher risk of mortality, mainly due to respiratory complications. In this study, 30-day mortality was $12.8 \%$ in patients with COVID-19 infection, which is a rate lower mortality than the report of other reviews $[5,6,8,9,13]$. Respiratory complications occurred in $30.8 \%$ of patients with COVID19 infection, whereas patients without COVID-19, only appeared in $1.4 \%$. This higher respiratory complication rate compares with other reports that have observed higher respiratory complications in patients with COVID-19 infection $[5,6,8,9,13]$. In the most significant study published to date by the CovidSurg group, the overall 30 -day mortality was $23.8 \%$, with $51.2 \%$ of respiratory complications and a mortality rate from respiratory complications of $38 \%$ [5]. Other studies report general mortality rates ranging from 12.5 to $83.3 \%$, with respiratory morbidity even up to $100 \%$ [6-15]. An Italian series of 41 patients infected with COVID-19 who underwent surgery describe overall mortality of $19.5 \%$ and respiratory morbidity of 59\% [8]. A retrospective study from Spain analyzed morbidity and mortality in patients operated on until the pandemic's peak in that country, reports mortality of $20 \%$ in COVID-19 patients and severe respiratory morbidity of $27 \%$ [13].

Similarly, a Chinese series that included 34 patients with a perioperative diagnosis of COVID-19 infection undergoing elective surgery reports a mortality rate of $20.5 \%$, requiring ICU management for respiratory failure in $44.1 \%$ of patients [6]. The available literature is summarized in Table 8 . The observed differences across studies can be attributed to a variety of factors such as the implemented protocols, the period and severity of the pandemic, variations in healthcare policies implemented by different countries, individuals between hospital centers and resources, to name a few.

Compared to the results reported in other series, our study's lower mortality and complication rates could be attributed to the fast and strict implementation of preventive measures within our institution from the beginning of the pandemic outbreak. In our opinion, the development of a multidisciplinary COVID-19 committee was essential for the development, implementation, and surveillance of 
Table 4 Postoperative outcomes

\begin{tabular}{|c|c|c|c|c|}
\hline & Total & COVID-19 (+) & COVID-19 (-) & $p$ value \\
\hline Patients & 701 & 39 & 662 & - \\
\hline \multicolumn{5}{|l|}{ 30-day mortality; $n(\%)$} \\
\hline Yes & $14(2 \%)$ & $5(12.8 \%)$ & $9(1.4 \%)$ & \multirow[t]{2}{*}{$<0.001$} \\
\hline No & $687(98 \%)$ & $34(87.2 \%)$ & $653(98.6 \%)$ & \\
\hline \multicolumn{5}{|c|}{ Respiratory mortality; $n(\%)$} \\
\hline Yes & $6(42.9 \%)$ & $5(100 \%)$ & $1(11.1 \%)$ & \multirow[t]{2}{*}{0.006} \\
\hline No & $8(57.1 \%)$ & $0(0 \%)$ & $8(88.9 \%)$ & \\
\hline \multicolumn{5}{|c|}{ Postoperative complications; $n(\%)$} \\
\hline Without complications & $601(85.7 \%)$ & $21(53.9 \%)$ & $580(87.6 \%)$ & \multirow[t]{3}{*}{$<0.001$} \\
\hline Clavien-Dindo I-II & $45(6.4 \%)$ & $8(20.5 \%)$ & $37(5.6 \%)$ & \\
\hline Clavien-Dindo $\geq$ III & $55(7.9 \%)$ & $10(25.6 \%)$ & $45(6.8 \%)$ & \\
\hline \multicolumn{5}{|c|}{ Respiratory morbidity; $n(\%)$} \\
\hline Yes & $21(3 \%)$ & $12(30.8 \%)$ & $9(1.4 \%)$ & \multirow[t]{2}{*}{$<0.001$} \\
\hline No & $680(97 \%)$ & $27(69.2 \%)$ & $653(98.6 \%)$ & \\
\hline \multicolumn{5}{|l|}{ IMV requirement } \\
\hline Yes & $8(38.1 \%)$ & $6(50 \%)$ & $2(22.2 \%)$ & \multirow[t]{2}{*}{0.401} \\
\hline No & $13(61.9 \%)$ & $6(50 \%)$ & $7(77.8 \%)$ & \\
\hline \multicolumn{5}{|l|}{ Readmission } \\
\hline Yes & $34(4.9 \%)$ & $8(20.5 \%)$ & $26(3.9 \%)$ & \multirow[t]{2}{*}{$<0.001$} \\
\hline No & $667(95.1 \%)$ & $31(79.5 \%)$ & $636(96.1 \%)$ & \\
\hline \multicolumn{5}{|l|}{ Readmission by cause } \\
\hline Medical & $16(47.1 \%)$ & $5(62.5 \%)$ & $11(42.3 \%)$ & \multirow[t]{2}{*}{0.551} \\
\hline Surgical & $18(52.9 \%)$ & $3(37.5 \%)$ & $15(57.7 \%)$ & \\
\hline \multicolumn{5}{|l|}{ Reoperation } \\
\hline Yes & $31(4.4 \%)$ & $2(5.1 \%)$ & $29(4.4 \%)$ & \multirow[t]{2}{*}{0.825} \\
\hline No & $670(95.6 \%)$ & $37(94.9 \%)$ & $633(95.6 \%)$ & \\
\hline
\end{tabular}

$I M V$ invasive mechanical ventilation

protocols aimed at minimizing the risk of in-hospital contagion between patients and healthcare professionals. Other measures included preventive isolation of patients, continuous training of personnel, mandatory use of personal protective equipment, rotating preventive quarantines of health teams, and restriction of visits to patients. Likewise, in all patients with or without respiratory symptoms, samples were obtained to detect COVID-19 upon admission. While the PCR result was pending, they were isolated and managed as COVID-19 (+) patients until confirmed or discarded.

There are few experiences reported in Latin America regarding surgical outcomes during the COVID-19 pandemic. Carpio Colmenares et al. published a Peruvian experience including 59 patients who underwent emergency abdominal laparoscopic surgery during 2020, and they compared this group of patients with a control group of patients who underwent surgery in 2019 [20]. They found no statistically significant differences regarding age, sex, duration of symptoms, the severity of surgical illness, and postoperative morbidity rate. Patients who underwent surgery during the COVID-19 pandemic had the most prolonged postoperative stay, and only one patient had a perioperative COVID-19 infection. The postoperative morbidity rate was $45.7 \%$ in the 2020 group, far higher than the $14.3 \%$ reported in our series; nevertheless, in our study, patients with both elective and emergency procedures were included, so both samples are not comparable. Gonzalez-Calatayud et al. [21] described their experience in a tertiary center in Mexico, including all patients with suspected or confirmed SARS-CoV-2 infection who underwent surgery. They report a mortality rate of $42.8 \%$, but they included patients undergoing a vast range of surgical procedures like C-section, tracheostomy, and general surgery procedures.

Finally, many Latin American countries had to face a lack of resources and deficient infrastructure during this pandemic. In Peru, for example, there is only one center 


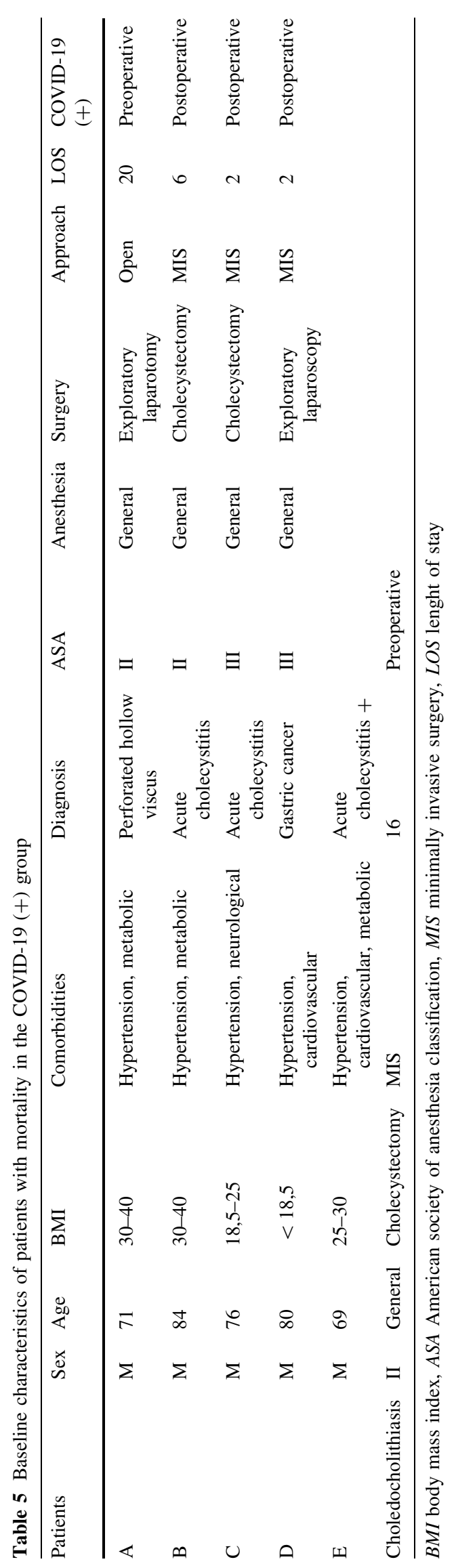


Table 6 Subgroup analysis of COVID-19 (+) patients

\begin{tabular}{llllll}
\hline Outcome & \multicolumn{2}{l}{ Preoperative COVID-19 $(n=20)$} & & \multicolumn{2}{c}{ Postoperative COVID-19 $(n=19)$} \\
\cline { 2 - 3 } & Symptomatic $(n=7)$ & Asymptomatic $(n=13)$ & & Symptomatic $(n=19)$ & Asymptomatic $(n=0)$ \\
\hline 30-day mortality & $2(10 \%)$ & $0(0 \%)$ & $0(0 \%)$ & $3(15.8 \%)$ & - \\
30-day morbidity & $5(25 \%)$ & $0(0 \%)$ & $13(68.4 \%)$ & - \\
Major complications* & $2(10 \%)$ & $0(0 \%)$ & $8(42.1 \%)$ & - \\
Respiratory complications & $4(20 \%)$ & $0(0 \%)$ & $8(42.1 \%)$ & - \\
IMV requirement & $2(10 \%)$ & $4(21.1 \%)$ & - \\
\hline
\end{tabular}

$I M V$ invasive mechanical ventilation

*Clavien-Dindo $\geq$ III

Table 7 Relative risk analysis

\begin{tabular}{|c|c|c|c|c|c|}
\hline Outcome & COVID-19 (+) & COVID-19 (-) & $\mathrm{RR}$ & $95 \% \mathrm{CI}$ & $p$ value \\
\hline 30-day mortality & $5 / 39$ & $9 / 662$ & 9.43 & $3.31-26.79$ & $<0.001$ \\
\hline 30-day mortality, excluding postoperative COVID & $2 / 20$ & $9 / 662$ & 7.35 & $1.69-31.86$ & 0.007 \\
\hline Major complications* & $10 / 39$ & $45 / 662$ & 3.77 & $2.06-6.90$ & $<0.001$ \\
\hline Major complications*, excluding postoperative COVID & $2 / 20$ & $45 / 662$ & 1.47 & $0.38-5.64$ & 0.573 \\
\hline
\end{tabular}

*Clavien-Dindo $\geq$ III

Table 8 Summary of available evidence of surgical outcomes during COVID-19 pandemic

\begin{tabular}{|c|c|c|c|c|c|c|}
\hline Author & Period (2020) & Total & $\begin{array}{l}\text { COVID-19 } \\
(+)\end{array}$ & $\begin{array}{l}\text { COVID-19 } \\
\text { mortality }\end{array}$ & Respiratory morbidity & $\begin{array}{l}\text { Major } \\
\text { complications* }\end{array}$ \\
\hline $\begin{array}{l}\text { COVIDSurg } \\
\text { collaborative }\end{array}$ & January-March & 1128 & 1128 & $23.8 \%(n=268)$ & $51.2 \%(n=577)$ & - \\
\hline Doglietto et al & February-April & 333 & 41 & $19.5 \%(n=8)$ & $58.5 \%(n=24)$ & $41.5 \%(n=17)$ \\
\hline Seretis et al & March-May & 100 & 3 & $0 \%$ & $66.6 \%(n=2)$ & - \\
\hline Lei et al & $\begin{array}{l}\text { January- } \\
\text { February }\end{array}$ & 34 & 34 & $20.5 \%(n=7)$ & $44.1 \%(n=15)$ & - \\
\hline Di Martino et al & February-March & 213 & 15 & $20 \%(n=3)$ & $33 \%(n=5)$ & $26.6 \%(n=4)$ \\
\hline Aminian et al & February & 4 & 4 & $50 \%(n=2)$ & $100 \%(n=4)$ & - \\
\hline Shrikhande et al & March-April & 494 & 6 & $0 \%$ & - & - \\
\hline Seeliger et al & March-May & 127 & 13 & $23 \%(n=3)$ & $61.5 \%(n=8)$ & $53.8 \%(n=7)$ \\
\hline Carpio Colmenares et al & March-June & 59 & 1 & $0 \%$ & - & $5.1 \%(n=3)$ \\
\hline $\begin{array}{l}\text { González-Catalayud } \\
\text { et al }\end{array}$ & April-July & 42 & 36 & $42.8 \%(n=18)$ & - & - \\
\hline
\end{tabular}

*Clavien-Dindo $\geq$ III

that has rooms with negative pressure equipment [22]. As a result, Latin American countries had difficulties achieving the requirements and guidelines set forth by professional societies from the rest of the world.

Concerning in-hospital COVID-19 infection, a study by Luong-Nguyen et al. reported that the risk augmented as the length of hospitalization increases has shown by $80 \%$ of in-hospital infection of COVID-19 in patients with hospital stay higher than 14 days (15-63 days), with general mortality of $13.3 \%$ [7]. These patients' infection could be directly related to hospital contagion, both by health personnel, support personnel, or patients accompanying them. In our study, COVID-19 infection was diagnosed during postoperative follow-up in 19 patients, $6(31.5 \%)$ of 
which occurred in patients with prolonged hospital stay between 20 to 87 days.

The cohort analyzed in this study included emergency operations as well as selected oncologic ones. None of the oncological patients in this cohort was deferred in their surgical treatment due to the pandemic. We report the case of a patient with severe COVID pneumonia, with thromboembolic events including a stroke during ICU hospitalization, who developed a first episode of rectorrhagia in the context of anticoagulation therapy. A colonoscopy detected a sigmoid adenocarcinoma (pT3N1bM0, IIIB staging). The oncological committee decided to defer the surgery until the patient was in better conditions during the hospital stay. A week later, the patient presented a second episode of lower GI bleeding with hemodynamic compromise. An emergency Hartmann surgery was performed. The patient recovered satisfactorily from surgery. Due to COVID-19 sequels and neurological sequels had prolonged hospitalization. The adjuvant treatment was deferred initially until the patient achieved a better performance status.

According to a subgroup analysis in the COVID-19 (+) group, those asymptomatic patients diagnosed prior to surgery (13 patients) had better outcomes, with no morbidity or mortality reported. In contrast, in symptomatic patients with preoperative infection (seven patients), five morbidities were diagnosed, four of them with respiratory morbidity, including two with IMV requirement who subsequently died by respiratory cause. In the group of COVID-19 (+) patients diagnosed in the postoperative follow-up (19 patients), all were symptomatic. Thirteen patients developed morbidities, eight had respiratory morbidities, four with IMV requirement, and three of them died by respiratory cause. Taking all this into account, we can say that asymptomatic patients with preoperative COVID19 infection were the ones with better outcomes, in contrast with those symptomatic in the preoperative or postoperative period that had the worst outcomes.

Our study has limitations and strengths. One limitation is that our cohort only included patients with esophagogastric, hepatobiliary, colorectal, or general abdominal surgeries, excluding procedures performed by other surgical specialties. Although this could lead to a selection bias, it allows us to analyze the perioperative results of a specific group of commonly performed procedures in surgical practice. Another limitation is the low prevalence of patients with COVID-19 infection compared to other series, which could also influence the lower rates of mortality and complications. The lower mortality and complications could also be attributed to the fact that our center is a private high complexity hospital. The availability of operation rooms, personal protection supplies, highly trained health care professionals, and specific isolation areas was only slightly compromised. Thus, critical care bed availability was maintained despite the high transitory demand during the pandemic's most critical periods.

Our results summarize the clinical outcomes of consecutive patients operated who underwent surgery during the first six months of the COVID-19 pandemic outbreak period in Chile. Various surgical services worldwide have adapted to minimize the risks in their patients [23, 24]. In this context, we provide our experience that can help others center to establish a decision-making process on surgical patients management during the pandemic. New studies and other experimental designs will be necessary to reliably determine the behavior and risk factors related to perioperative infection by COVID-19.

In conclusion, 30-day mortality, specific respiratory mortality, and major surgical complications are higher in patients with perioperative COVID-19 infection. The observed lower rate of mortality and complications than the reported in other studies could be explained to the implementation and fulfillment of strict systematized protocols for preventive isolation, protection of personnel, preventing isolation of patients, and PCR screening of all patients admission to discharge. As more countries are facing the second wave of COVID-19 infection, we hope that the lessons learned during the first wave will help to improve surgical outcomes during the ongoing current COVID-19 pandemic.

Funding This study did not receive any funding.

\section{Declarations}

Conflict of interest The authors declare that they have no conflict of interest.

Ethical approval The institutional ethics committee approved the present study.

\section{References}

1. Surgeons (2020) ACo local resumption of elective surgery guidance. American College of Surgeons

2. Departamento de Epidemiología $\mathrm{MdS} 45^{\circ}$ Informe Epidemiológico Enfermedad por COVID-19, Santiago de Chile, MINSAL, 2020

3. Besnier E, Tuech JJ, Schwarz L (2020) We asked the experts: covid-19 outbreak: is there still a place for scheduled surgery? "Reflection from pathophysiological data". World J Surg 44:1695-1698. https://doi.org/10.1007/s00268-020-05501-6

4. Huang C, Wang Y, Li X et al (2020) Clinical features of patients infected with 2019 novel coronavirus in Wuhan, China. Lancet 395:497-506

5. Collaborative C (2020) Mortality and pulmonary complications in patients undergoing surgery with perioperative SARS-CoV-2 infection: an international cohort study. Lancet 396:27-38 
6. Lei S, Jiang F, Su W et al (2020) Clinical characteristics and outcomes of patients undergoing surgeries during the incubation period of COVID-19 infection. EClinicalMedicine 21:100331

7. Luong-Nguyen M, Hermand H, Abdalla S et al (2020) Nosocomial infection with SARS-Cov-2 within departments of digestive surgery. J Visc Surg 157:S13-S18

8. Doglietto F, Vezzoli M, Gheza F et al (2020) Factors associated with surgical mortality and complications among patients with and without coronavirus disease 2019 (COVID-19) in Italy. JAMA Surg 155(8):691-702

9. Aminian A, Safari S, Razeghian-Jahromi A et al (2020) COVID19 outbreak and surgical practice: unexpected fatality in perioperative period. Ann Surg 272:e27-e29

10. Cai M, Wang G, Zhang L et al (2020) Performing abdominal surgery during the COVID-19 epidemic in Wuhan, China: a single-centred, retrospective, observational study. Br J Surg 107:e183-e185

11. Fu D, Zhang P, Wang L et al (2020) Emergency abdominal surgery in COVID-19 patients: a note of caution from Wuhan. $\mathrm{Br}$ J Surg 107:e262

12. McDermott A, O'Kelly J, de Barra E et al (2020) Perioperative outcomes of urological surgery in patients with SARS-CoV-2 infection. Eur Urol 78:118-120

13. Di Martino M, Septiem JG, González RM, Nova JL, Rodríguez Hoz, Bonito AC, Martín-Pérez E (2020) Elective surgery during the SARS-CoV-2 pandemic (COVID-19): morbidity analysis and recommendations on patient prioritization and safety measures. Span Surg 98(9):525-32

14. Gallego MÁ, de las Casas SG, Migueláñez IP, Rubio-Pérez I, Serrano CB, Peña EÁ, Domínguez JD (2020) Impacto de la pandemia por SARS-CoV-2 sobre la actividad y profesionales de un Servicio de Cirugía General y del Aparato Digestivo en un hospital terciario. Cir Esp 98(6):320-7

15. Pérez-Rubio Á, Sebastián Tomás JC, Navarro-Martínez $\mathrm{S}$ et al (2020) Incidence of surgical abdominal emergencies during SARS-CoV-2 pandemic. Cir Esp 98(10):618-24

16. Yang Y, Yang M, Shen C et al (2020) Evaluating the accuracy of different respiratory specimens in the laboratory diagnosis and monitoring the viral shedding of 2019-nCoV infections. MedRxiv 78(3):241

17. Dindo D, Demartines N, Clavien PA (2004) Classification of surgical complications: a new proposal with evaluation in a cohort of 6336 patients and results of a survey. Ann Surg 240:205-213

18. Irarrázaval MJ, Inzunza M, Muñoz R et al (2020) Telemedicine for postoperative follow-up, virtual surgical clinics during COVID-19 pandemic. Surg Endosc. https://doi.org/10.1007/ s00464-020-08130-1

19. Pattillo JC, Rabagliati R (2020) Atención de pacientes confirmados COVID-19, casos sospechosos COVID-19 o contacto de alto riesgo en pabellón. Santiago de Chile, Red Salud UCCHRISTUS

20. Carpio Colmenares YT, Ruiz Cárdenas, de Castilla D, García Barrionuevo LA et al (2020) Emergency abdominal laparoscopic surgery during the coronavirus disease 2019 pandemic: experience in a private center in Peru. J Laparoendosc Adv Surg Tech A 31(3):261-265

21. González-Calatayud DM, Vargas-Ábrego DB, Gutiérrez-Uvalle DGE et al (2020) Observational study of the suspected or confirmed cases of sars COV-2 infection needing emergency surgical intervention during the first months of the pandemic in a third level hospital: case series. Ann Med Surg (Lond) 60:149-154

22. Panduro-Correa V, Arteaga-Livias K, Rodríguez-Morales AJ (2020) Coronavirus disease 2019 (COVID-19) and surgical recommendations in latin America. Am Surg 86:596-598

23. Elizabeth Brindle M, Gawande A (2020) Managing COVID-19 in surgical systems. Ann Surg 272:e1-e2

24. Heffernan DS, Evans HL, Huston JM et al (2020) Surgical infection society guidance for operative and peri-operative care of adult patients infected by the severe acute respiratory syndrome coronavirus-2 (SARS-CoV-2). Surg Infect (Larchmt) 21:301-308

Publisher's Note Springer Nature remains neutral with regard to jurisdictional claims in published maps and institutional affiliations. 